\title{
ON MAGNETIC ACTIVITY BAND OVERLAP, INTERACTION, AND THE FORMATION OF COMPLEX SOLAR ACTIVE REGIONS
}

\author{
SCOTt W. McIntosh ${ }^{1,2}$ AND Robert J. LeAmon ${ }^{3}$ \\ ${ }^{1}$ High Altitude Observatory, National Center for Atmospheric Research, P.O. Box 3000, Boulder, CO 80307, USA; mscott@ hao.ucar.edu \\ ${ }^{2}$ School of Mathematics and Statistics, University of St Andrews, St Andrews, Fife KY16 9SS, UK \\ ${ }^{3}$ Department of Physics, Montana State University, Bozeman, MT 59717, USA \\ Received 2014 September 7; accepted 2014 October 23; published 2014 November 10
}

\begin{abstract}
Recent work has revealed a phenomenological picture of the how the $\sim 11 \mathrm{yr}$ sunspot cycle of the Sun arises. The production and destruction of sunspots is a consequence of the latitudinal-temporal overlap and interaction of the toroidal magnetic flux systems that belong to the $22 \mathrm{yr}$ magnetic activity cycle and are rooted deep in the Sun's convective interior. We present a conceptually simple extension of this work, presenting a hypothesis on how complex active regions can form as a direct consequence of the intra- and extra-hemispheric interaction taking place in the solar interior. Furthermore, during specific portions of the sunspot cycle, we anticipate that those complex active regions may be particularly susceptible to profoundly catastrophic breakdown, producing flares and coronal mass ejections of the most severe magnitude.
\end{abstract}

Key words: magnetic fields - stars: activity - Sun: activity - Sun: evolution - Sun: interior - sunspots

Online-only material: color figures

\section{INTRODUCTION}

Recent work has exploited a novel analysis method for line-of-sight magnetograms called the "magnetic range of interaction" (McIntosh et al. 2007) to illustrate the presence of the elusive giant convective scale (e.g., Beck et al. 1998; Hathaway et al. 2013) in the photosphere and to present markers of that scale (McIntosh et al. 2014c). The most familiar of these markers to the reader may be the (ubiquitous) coronal brightpoint (e.g., Golub et al. 1974), although their potential association with the deep convective interior may have gone largely unnoticed.

Following that discovery, McIntosh et al. (2014d, hereafter Paper I) presented the temporal evolution of the markers of the giant scale and coronal brightpoints. They deduced the landmarks and phases of the sunspot cycle: the ascending phase, solar maximum, the declining phase, and solar minimum arise as a result of the latitudinal-temporal interaction of (toroidal) magnetic flux systems (or what we will refer to hereafter as "activity bands") that belong to the 22 yr magnetic activity cycle. In short, the appearance of the sunspot cycle could be explained in terms of the destructive "interference" of these oppositely signed activity bands as they migrate equatorward.

The main result of Paper I is illustrated in panel (B) of Figure 1. In short, the temporal and latitudinal interaction of the oppositely signed activity bands in each hemisphere, and across the equator, modulate the occurrence of sunspots (on the low-latitude pair). We noticed that activity bands of the same sign appear at high latitudes $\left(\sim 55^{\circ}\right)$ every $22 \mathrm{yr}$ and migrate equatorward, taking approximately $19 \mathrm{yr}$ to reach the equator. The low-latitude pair of bands abruptly "terminates" at the equator. This equatorial termination, or cancelation, signals the end of one sunspot cycle and leaves only the higher-latitude band in each hemisphere. For example, the cycle 23 sunspots did not appear to grow in abundance (or area) until the cycle 22 bands had terminated in mid-1997; likewise, in early 2011, the growth of cycle 24 sunspots followed the termination of the cycle 23 bands. Sunspots rapidly appeared and grew on that band for several years until the next (oppositely signed) band appeared at high latitude, a relationship that was determined empirically in Paper I and that defines the maximum activity level of the new cycle by triggering a downturn in sunspot production on the low-latitude band. The perpetual interaction of these temporally offset $22 \mathrm{yr}$ activity bands drives the quasi $11 \mathrm{yr}$ cycle of sunspots which forms the envelope of the Sun's magnetically driven activity where the degree of overlap in the bands appears to inversely govern our star's sunspot production (more overlap, less spots and vice versa). This observational evidence presented in Paper I points to the rotational energy at the bottom of our star's convective interior as being either a major driver or the major driver of its long-term (magnetic) evolution, where we suppose that perturbations to that system and the sunspots that those perturbations produce have the potential to impact shortterm variability.

In this Letter we present an extension of the activity band overlap and interaction concept that was introduced in Paper I. We consider the formation of complex active regions (Figure 2) in light of this picture, where and when these regions might form, and their potential to form delta regions - the subset of active regions responsible for the vast majority of $\mathrm{M}$ - and $\mathrm{X}$-class flares (e.g., Benz 2008). It is then a further extension to consider epochs when the most catastrophic flares that give rise to the most intense geomagnetic storms, i.e., those of a magnitude similar to the 1859 September 1 "Carrington event" (Carrington 1859), have a higher probability of occurrence.

\section{BAND-BAND INTERACTION}

Figure 3 builds upon the activity bands of Figure 1 to illustrate the spatio-temporal zones where we would expect to see signatures of band-band interaction occur naturally, here drawn as pink and green hatched ovals. The pink ovals represent "simple" mixing. In those zones, the erupting magnetic flux could come from either polarity of the band or a mix (in the interior) before emerging to create what would appear to be an "anomalous" active region with characteristics of both the 
(A) ROB/SIDC Monthly Hemispheric Sunspot Number

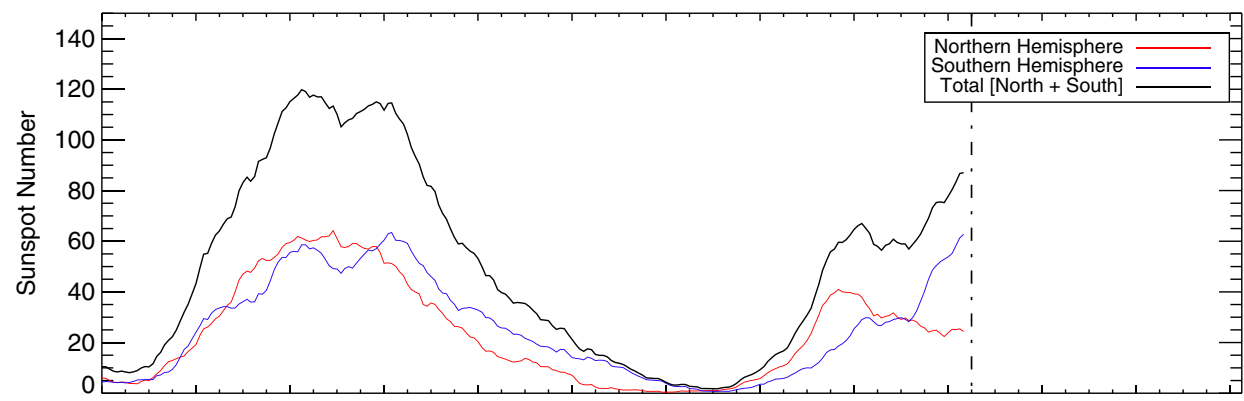

(B) Data-Derived SOHO/SDO Solar Cycle Band-O-Gram

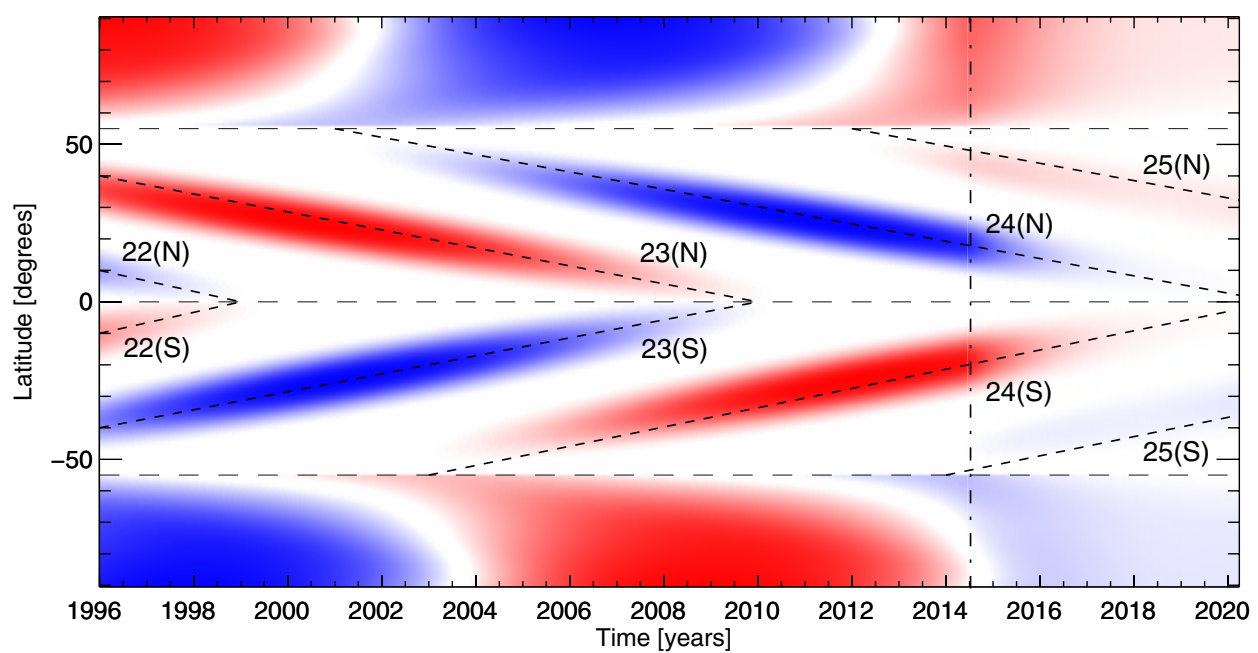

Figure 1. Panel (A) shows the total and hemispheric sunspot numbers for cycles 23 and 24 as black, red, and blue solid lines, respectively. Panel (B) shows the data-determined schematic of the migrating activity bands that belong to the $22 \mathrm{yr}$ magnetic activity cycle from McIntosh et al. (2014d). The interplay of these bands gave rise to sunspot cycles 22-24 as indicated. In each case, the color of the band reflects its polarity (red is positive; blue is negative). The dashed horizontal lines indicate the equator and lines of the 55th parallel in each hemisphere while the vertical dot-dashed lines indicate the current time. The reader will notice that, in addition to the cycle 25 band's appearance between 2012 and 2014, we have attempted to indicate the progression of the activity bands into the future as outlined in McIntosh et al. (2014d). This projection does not carry information about the strength of activity on the bands in the future, only their migratory paths.

(A color version of this figure is available in the online journal.)

current and upcoming cycles, but this would be many years before the true spots and active region complexes of the latter appeared. Also, the active region pairs at high latitude would have the same helicity given that the bands from which the flux originates are in the same hemisphere-left-handed in the north, right-handed in the south (Richardson 1941; Zhang et al. 2010a). One should note that this process can only occur after the new cycle bands at high latitude have started their equatorward migration-by our earlier declaration, this is after solar maximum in the declining phase of the sunspot cycle for each hemisphere. So, we would expect the frequency of mixed, or reversed, active region complexes to increase significantly in the declining phase, occurring on the high-latitude periphery of the butterfly wings (the tear-shaped areas enclosing the hemispheric sunspots of each cycle). From a space weather climatology standpoint, we would be able to watch these zones carefully for such flux systems and be able to monitor how they interact with their surroundings.

The green cross-hatched ovals indicate a second zone, an equatorial mixing zone, where the bands of the dying cycle interact at low latitudes across the equator. These bands will have opposite polarity and opposite helicity since they are anchored in different hemispheres and experience different signs of the Coriolis force. These active region complexes would occur on the equatorward periphery of the butterfly wings in the deep descending phase of one sunspot cycle and into the early ascending phase of the next. We anticipate that the crosspolarity, opposite-helicity mixing of the low-latitude activity bands should produce violent active region complexes-as demonstrated by studies of flux system interaction (e.g., Linton et al. 2001; Archontis et al. 2007). Again, from a forecast standpoint, we would know roughly where and when to look for such regions as they emerge.

Figure 4 uses the results published by Harvey (1992) that were extracted from the detailed studies of the magnetic configurations of active regions in cycles 20-22 by Howard (1989, 1991). To guide the reader, we have estimated the positions of the activity bands in this butterfly diagram using the same technique used to create Figures 11 and 12 in Section 5 of Paper I. In short, we use the hemispheric sunspot maxima to determine the start time of band migration at high latitude and the abrupt increase of spot area (on the high-latitude new cycle bands) to estimate the time of termination for the low-latitude bands. Together, these landmarks allow us to linearly estimate where the activity band would be. One should note that the vast majority of points in the plot (the crosses) have the "correct orientation" in that they appear "as expected" for that cycle, to use the language of Harvey (1992). The open circles, on the other hand, represent anomalous active regions, those where the footprints of the system have the "reversed orientation" from what was 


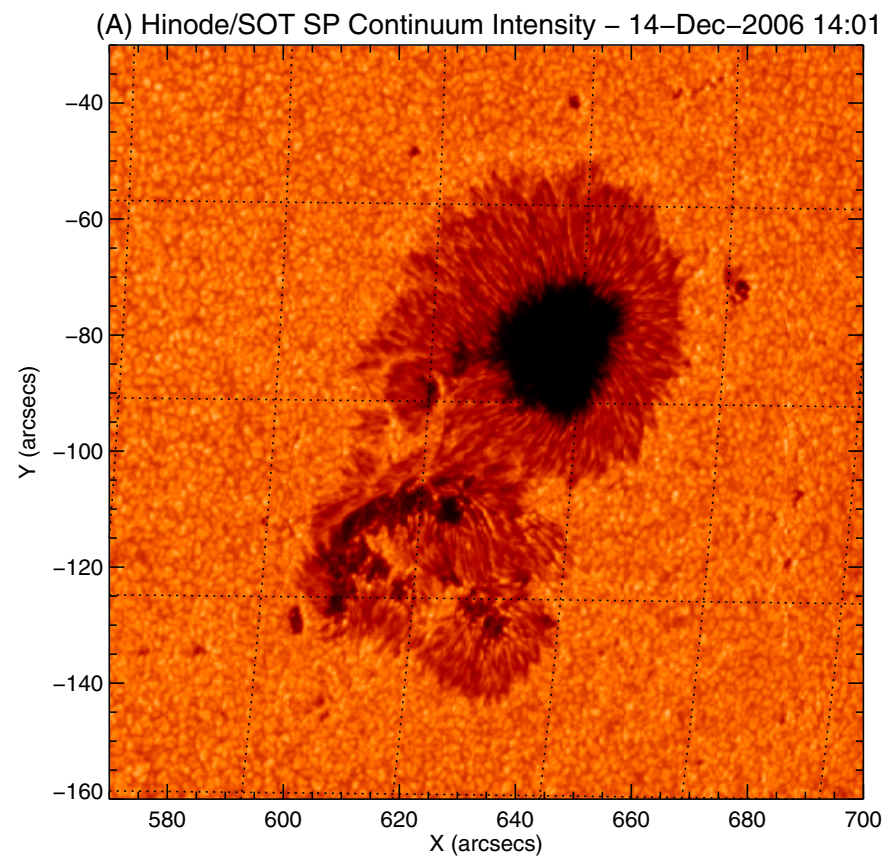

(B) HAO/CSAC Inverted B - 14-Dec-2006 14:01

Figure 2. Hinode/Solar Optical Telescope (SOT) Spectropolarimeter observations of NOAA AR 10930, a complex active region ( $\beta \gamma \delta$-Mount Wilson; Dki-McIntosh classification) near the solar equator on 2006 December 14. Panel (A) shows the continuum intensity neighboring the Fe I $6302.5 \AA$ A line and panel (B), the line-of-sight magnetogram inferred from a Milne-Eddington inversion of the measured Stokes profiles. This active region fired the final significant salvos of solar cycle 23, including four X-class flares, as it traversed the Earth-facing side of the Sun.

(A color version of this figure is available in the online journal.)

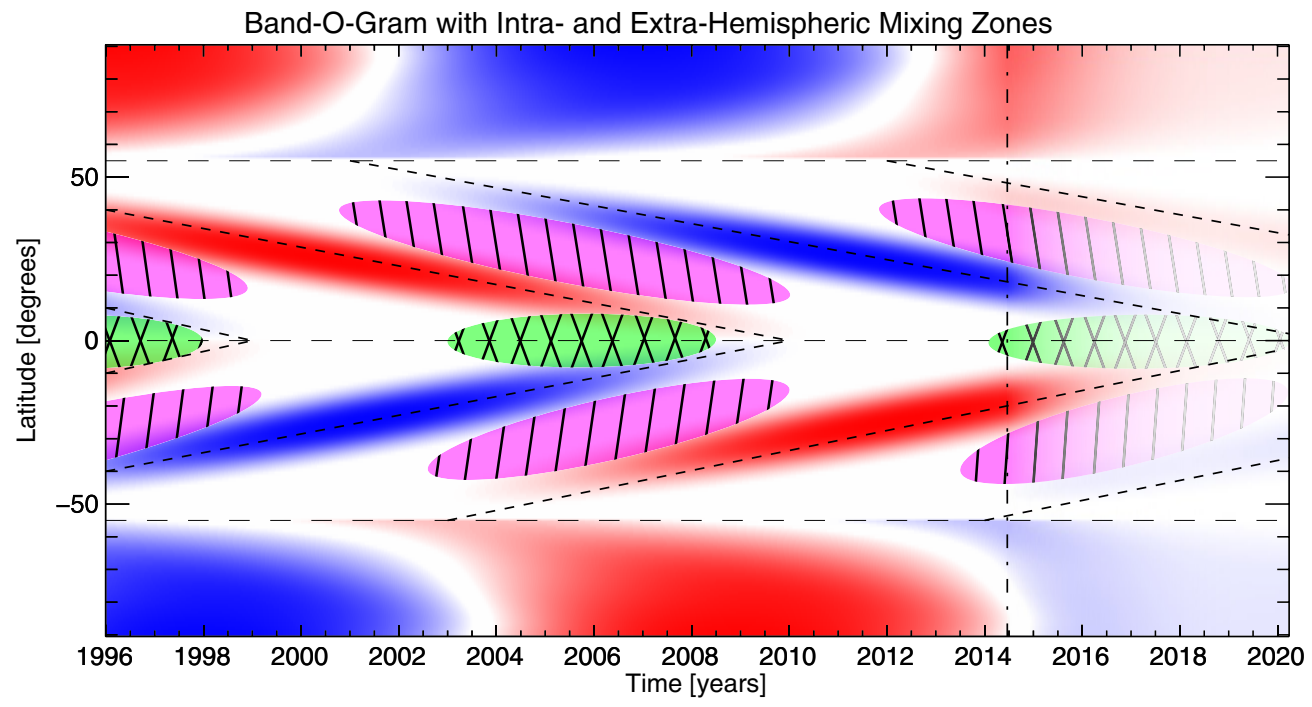

Figure 3. Data-determined schematic of the migrating activity bands that belong to the $22 \mathrm{yr}$ magnetic activity cycle from McIntosh et al. (2014d)—compare with Figure 1. In addition to the forecast, we have now estimated the latitudes and times for which cross-band mixing becomes important—-these regions are drawn as pink and green ellipses (see text). The main distinguishing factor between these two interaction regions is the impact that helicity can have on them and so the green ellipses around the equator are cross-hatched to indicate the potential for cross-band and opposite-helicity mixing across the solar equator.

(A color version of this figure is available in the online journal.)

expected. Many of those open circles appear in locations that we would have expected given the interacting flux-band picture introduced in Paper I and drawn in Figure 3 above.

\section{1. "Delta" Regions}

Returning to an analysis of contemporary data, Figure 5 presents a comparison between the activity band interaction schematic of Figure 1, the hemispheric and total sunspot numbers (with corresponding numbers of delta regions), and the latitude-time distribution of active regions (black $\triangle$ ) and regions with a delta classification (red $\circ$ ). Locations are taken from the
US Air Force ${ }^{4}$ active region record. The textbook definition of a "delta" region as two oppositely signed umbrae (separated by less than $2^{\circ}$ ) within one penumbra traces back to Hale et al. (1919) and Künzel (1960), who added the delta classification to Hale's original $\alpha, \beta$, and $\gamma$ sunspot classifications. As discussed above, these delta regions, which compose only a few percent of the total number of sunspots, are the subset responsible for the vast majority of M- and X-class flares (e.g., Benz 2008).

\footnotetext{
4 The USAF sunspot record can be retrieved from Dr. David Hathaway at
} NASA/MSFC (http://solarscience.msfc.nasa.gov/greenwch.shtml). 


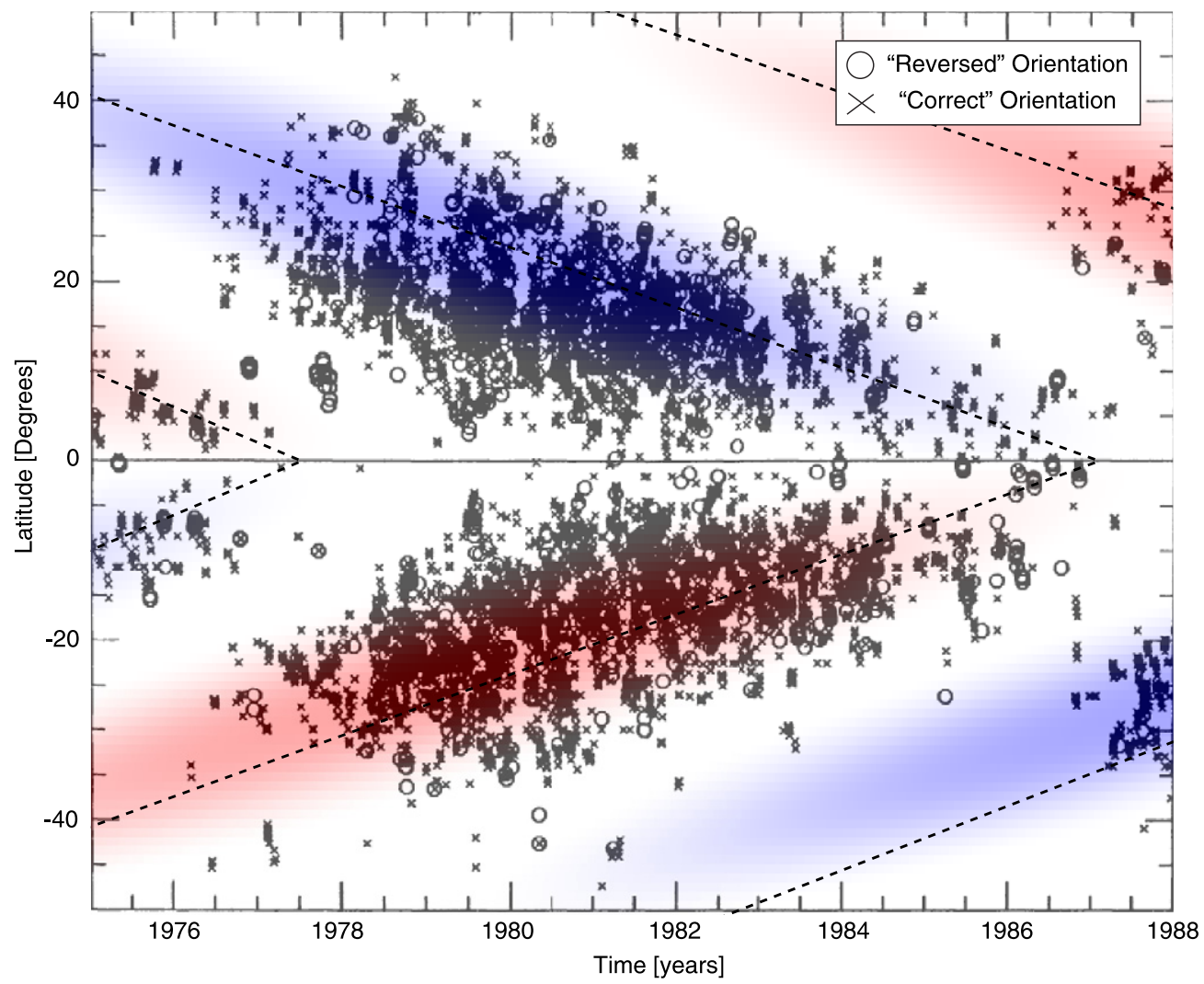

Figure 4. Contrasting the orientation of magnetic bipolar regions in a latitude-time diagram of "properly oriented" (x) and "reverse oriented" ( $)$ ) bipolar regions from sunspot cycles 20-22 (Harvey 1992). Overlaid are estimates of the $22 \mathrm{yr}$ magnetic activity band locations that modulate those sunspot cycles (see Figure 1). The extracted figure and data points of Harvey (1992) are reproduced with permission of PASJ. Figure 3 of Harvey, Karen L. The Solar Cycle; Proceedings of the National Solar Observatory/Sacramento Peak 12th Summer Workshop, ASP conference series, vol. 27 (San Francisco, CA: ASP), 335.

(A color version of this figure is available in the online journal.)

(A) Monthly Sunspot And Delta Spot Frequency

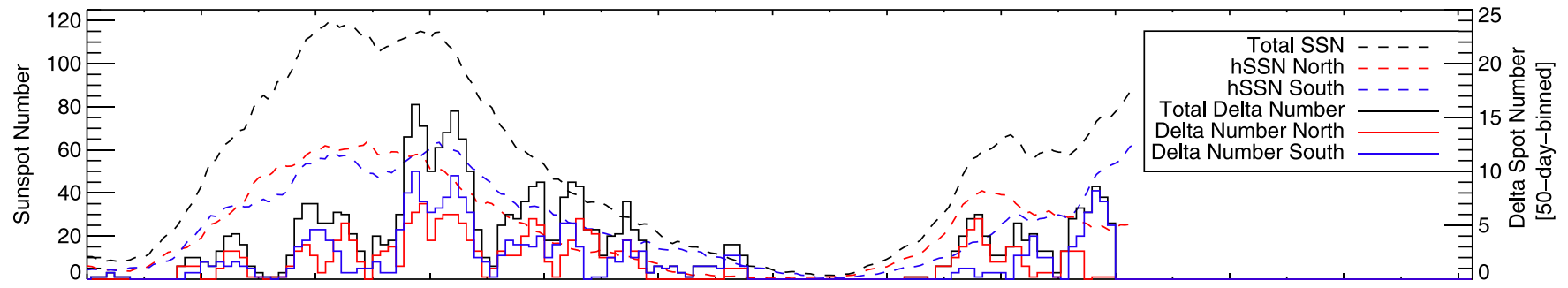

(B) USAF Sunspot Butterfly Diagram

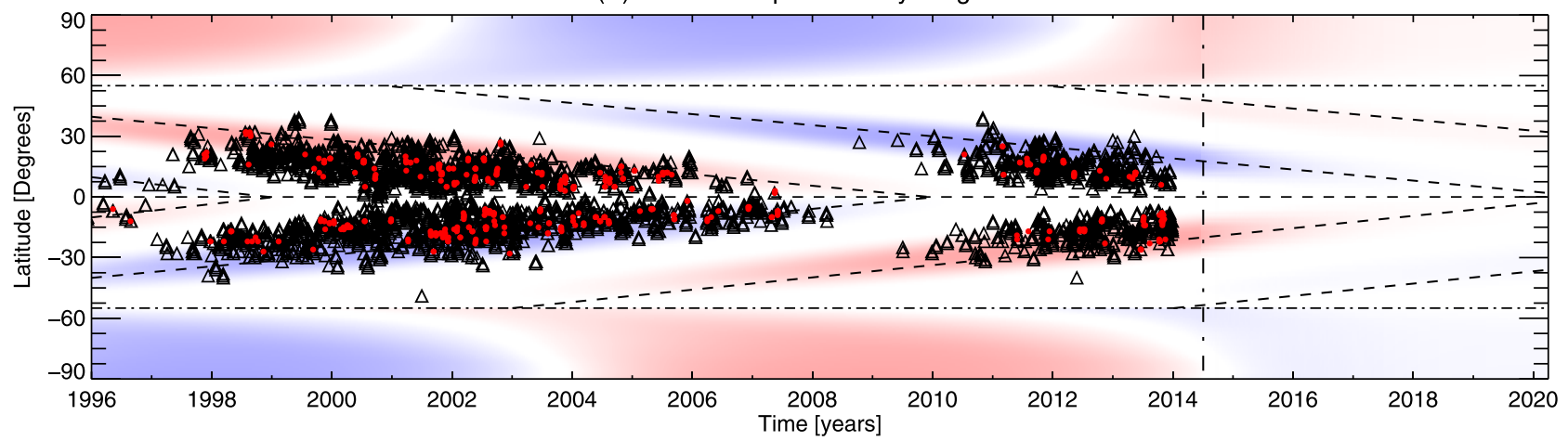

Figure 5. (a) Comparing the number of delta-classified regions (black solid line) with the hemispherically decomposed number of the same (red is north; blue is south) vs. the corresponding total and hemispheric (monthly) sunspot numbers. (b) Comparing regular (black $\triangle$ ) and delta-classified region (red $\circ$ ) locations for solar cycles 23 and 24 from the US Air Force active region record. These locations are overlaid on the activity bands shown in Figures 1 and 3.

(A color version of this figure is available in the online journal.) 
Therefore, their origins are critically important in the context of space weather.

Comparing Figure 5 with our earlier analysis and discussion of Figure 3, we see that the delta-classified regions appear throughout the active region butterfly diagram, but with a possible preference of a drift from the high-latitude to lowlatitude periphery of the butterfly diagram. In the deepest part of the descending phase-from $\sim 2004$ on-the delta-classified regions tend to appear predominantly on the low-latitude periphery of the butterfly in each hemisphere. These delta regions could be born of extra-hemispheric band interaction where bands of opposite polarity and opposite helicity can mix. Further, considering the total and hemispheric delta numbers for cycle 23 (panel (a)), we see that the number of delta-classified regions appears to be weighted to the descending phase of the cycle. We also notice an apparent clustering (in time) of regions that, when considered as a time series, create a quasi-periodicity in the delta region numbers of each hemisphere (and the total). The periodicity and timing of the peaks in the delta region time series match other proxies of magnetic solar activity that have been tied to significant surges of magnetic flux emergence from the deep interior that are of unknown physical origin (McIntosh et al. 2014b). An extended study of this pattern is beyond the scope of this Letter, but an effort in preparation (Leamon et al. 2015) will use the Mt. Wilson activity and sunspot record (e.g., Pevtsov et al. 2013) back to the early 20th century to explore these relationships.

\section{DISCUSSION}

In the above analysis we have noted that the band-band interaction hypothesis of Paper I can be used to explain the occurrence of some, but not all, reversed or irregular sunspot groups and active regions. The band-band interaction scheme that we have discussed above would lead us to deduce that the occurrence rate of such regions (in each hemisphere) should grow after the sunspot maximum (in each hemisphere). This is a time when there are two activity bands in each hemisphere. The interaction regions between the activity bands should be preferred locations for irregular active regions to form. This appears to be supported by the evolution shown in Figure 4 (and Harvey 1992). The equatorial interaction region is of particular interest in the context of space weather. In this region, it is possible that activity bands of mixed sign and helicity can interact with each other across the equator. The analysis of Figure 5 would tend to support that deduction.

In addition to the declaration of irregular, reversed, or Hale'slaw-violating active regions (Hale 1924), there are numerous "anomaly" reports in the literature which would tend to support our hypothesis and lend greater support to the band-band interaction concept as a key framer of solar activity. These anomalies include very high-latitude sunspot groups after solar maximum (Carrington 1860, just as the next bands appear), regions of incorrectly sensed magnetic helicity showing up in the wrong hemisphere around the equator (Zhang et al. 2010a, 2010b; Zhang 2012; Liu et al. 2014), sunspots with incorrectly oriented super-penumbral whorls (e.g., Richardson 1941; Peter 1996; Balasubramaniam et al. 2004), and X-ray sigmoids of the wrong shape in the wrong hemisphere (e.g., Rust \& Kumar 1996; Leamon et al. 2002; Canfield et al. 2007).

Indeed, while neither Rust \& Kumar (1996) nor Leamon et al. (2002) found lockstep agreement between hemisphere and sigmoid helicity handedness, the greater preponderance of "correctly" kinked sigmoids $(\sim 4$ in 5 by the former, compared to "only" $\sim 2$ in 3 to the latter) can be explained by when the data were collected for the two studies. Rust \& Kumar (1996) focused on just 15 months (1991 October-1993 January) at solar maximum when our band hypothesis would imply there is only the single flux system present, whereas Leamon et al.'s data set covered the entire descending phase of cycle 22 and the early rise phase of cycle 23 when, as we have seen, band-band interactions would favor the appearance of mixed-helicity active regions. However, a more detailed study is required to identify such regions in the full historical record and investigate if these reported anomalies really are a very natural by-product of this complex band-band interaction taking place in the solar interior.

\subsection{The Origin of Extreme (Space Weather) Events?}

Extending our deductive reasoning, we consider the occurrence of the most profound of solar events. These solar storms (e.g., Carrington 1859) would appear at first glance to occur randomly - the vast majority do not occur at sunspot maxima (e.g., Odenwald et al. 2006). Based on our earlier deduction that (devastatingly) complex active regions can occur in the equatorial interaction region where cross-polarity and opposite-helicity bands can interact, we would naturally expect a bias toward times of activity band overlap - the deep declining phase and early ascending phase of solar activity (see Figure 1). Therefore, we deduce that the length of time spent by the activity bands at very low latitudes is strongly related to the probability that a devastating solar storm will occur. In Paper I, it was discussed that the number of sunspots produced in a given cycle was inversely related to the degree of overlap between the interacting bands. Shorter overlap times tend to produce a long ascending phase but a short and strong cycle, while longer overlap times produce shorter ascending phases and weaker, longer cycles. McIntosh et al. (2014a) discusses a mode where the overlap of the activity bands is at its greatest possible extent-one where there may be no sunspots produced for an extended period of time- - a grand (sunspot) minimum - even though the Sun would continue to cycle. It is very likely that these very lengthy overlaps, times of prolonged lows in sunspot numbers, are significantly more likely to produce an event of the scale of that witnessed by Carrington (1859). Unfortunately, the full record of such extreme events is limited by the fact that we "catch" only those that are geo-effective, thus creating a selective bias in the sample required to fully test our hypothesis. A forthcoming study by Leamon et al. (2015) will consider this topic at greater length as it has considerable potential impact on our ability to foretell potentially cataclysmic solar storms.

\section{CONCLUSION}

The discussion presented above logically extends the concept presented in Paper I of how the activity bands that belong to the $22 \mathrm{yr}$ magnetic activity cycle (phenomenologically) explain the landmarks of the sunspot cycle to complex active regions and delta regions. We deduce that such complex regions form as a consequence of the intra- and extra-hemispheric interactions taking place in the solar interior between those bands-this interaction can naturally result in active regions of mixed polarity and built-in complexity before emergence into the outer atmosphere. However, we acknowledge that many physical processes internal to the activity band could also produce "irregular" sunspots and active regions (e.g., Archontis et al. 2007; Pevtsov et al. 2013; Liu et al. 2014). Further, we have deduced that a class of such complex active regions can exhibit 
interaction from flux systems of mixed helicity in addition to polarity. Those regions most frequently would occur in the deep descending phase of sunspot cycles and present a significant threat to much of the solar system as they can explosively release their (stored) magnetic energy (Linton et al. 2001) in close proximity to the solar equator. When these near-equatorial bands remain in close proximity for extended times, as one may expect on the entry into grand minima (McIntosh et al. 2014a), the likelihood of a profound eruptive event would increase.

We acknowledge that while compelling, this is but one way to generate complex active regions and delta spots. Another way is that new flux tubes from the same band offset in latitude or longitude (or depth) could be sufficiently buffeted by turbulent motions deep below the photosphere so as to emerge into existing systems, giving the appearance of an incorrectly oriented system.

Nevertheless, the hypothesis presented here points to a picture of the Sun's deep convective interior, and the magnetic interactions taking place therein, which is not yet accessible to numerical simulation although we note that steps in that direction have been taken (e.g., Weber et al. 2012; Nelson et al. 2014). Such investigations are necessary to develop the considerable forecast skill required to protect Earth- and space-based infrastructure required for our technologically driven society.

NCAR is sponsored by the National Science Foundation. We acknowledge support from NASA contracts NNX08BA99G, NNX11AN98G, NNM12AB40P, NNG09FA40C (IRIS), and NNM07AA01C (Hinode). Hinode SOT/SP Inversions were conducted at NCAR under the framework of the Community Spectropolarimtetric Analysis Center ([http://www.csac.hao. ucar.edu/]CSAC). We also thank Alan Title, Giuliana de Toma, Fraser Watson, and Luca Bertello for the discussion that formed the basis of a deeper (historical) investigation to follow.

\section{REFERENCES}

Archontis, V., Hood, A. W., \& Brady, C. 2007, A\&A, 466, 367

Balasubramaniam, K. S., Pevtsov, A., \& Rogers, J. 2004, ApJ, 608, 1148

Beck, J. G., Duvall, T. L., \& Scherrer, P. H. 1998, Natur, 394, 653

Benz, A. O. 2008, LRSP, 5, 1

Canfield, R. C., Kazachenko, M. D., Acton, L. W., et al. 2007, ApJL, 671, L81

Carrington, R. C. 1859 , MNRAS, 20, 13

Carrington, R. C. 1860, MNRAS, 20, 254

Golub, L., Krieger, A. S., Silk, J. K., Timothy, A. F., \& Vaiana, G. S. 1974, ApJL, 189, L93

Hale, G. E. 1924, PNAS, 10, 53

Hale, G. E., Ellerman, F., Nicholson, S. B., \& Joy, A. H. 1919, ApJ, 49, 153

Harvey, K. L. (ed.) 1992, in ASP Conf. Ser. 27, The Solar Cycle (San Francisco, CA: ASP), 335

Hathaway, D. H., Upton, L., \& Colegrove, O. 2013, Sci, 342, 1217

Howard, R. F. 1989, SoPh, 123, 271

Howard, R. F. 1991, SoPh, 135, 43

Künzel, H. 1960, AN, 285, 271

Leamon, R. J., Canfield, R. C., \& Pevtsov, A. A. 2002, JGRA, 107, 1234

Leamon, R. J., McIntosh, S. W., Watson, F. T., et al. 2015, ApJ, submitted

Linton, M. G., Dahlburg, R. B., \& Antiochos, S. K. 2001, ApJ, 553, 905

Liu, Y., Hoeksema, J. T., \& Sun, X. 2014, ApJL, 783, L1

McIntosh, S. W., Davey, A. R., Hassler, D. M., et al. 2007, ApJ, 654, 650

McIntosh, S. W., Leamon, R. J, \& Centeno-Elliott, R. 2014a, Frontiers Space Sci., submitted

McIntosh, S. W., Leamon, R. J, Krista, L. D., et al. 2014b, NatCo, in press (https://dl.dropboxusercontent.com/u/3057160/McIntoshSWx_Submit.pdf)

McIntosh, S. W., Wang, X., Leamon, R. J., \& Scherrer, P. H. 2014c, ApJL, 784, L32

McIntosh, S. W., Wang, X., Leamon, R. J., et al. 2014d, ApJ, 792, 12

Nelson, N. J., Brown, B. P., Sacha Brun, A., Miesch, M. S., \& Toomre, J. 2014, SoPh, 289, 441

Odenwald, S., Green, J., \& Taylor, W. 2006, AdSpR, 38, 280

Peter, H. 1996, MNRAS, 278, 821

Pevtsov, A. A., Bertello, L., Tlatov, A. G., et al. 2013, SoPh, 289, 593

Richardson, R. S. 1941, ApJ, 93, 24

Rust, D. M., \& Kumar, A. 1996, ApJL, 464, L199

Weber, M. A., Fan, Y., \& Miesch, M. S. 2012, SoPh, 287, 239

Zhang, H. 2012, MNRAS, 419, 799

Zhang, H., Sakurai, T., Pevtsov, A., et al. 2010a, MNRAS, 402, L30

Zhang, H., Yang, S., Gao, Y., et al. 2010b, ApJ, 719, 1955 